Editorial

\title{
Expressed citrus genome: integration is the challenge
}

When the CitEST project - 'Integration of breeding, comparative and functional genomics of citrus' was submitted to $\mathrm{CNPq}$ as a "Millennium Institute" proposal, the group of researchers already had a perception of how challenging such a proposal could be. First, the integration of genomic data with genetics and breeding in a woody perennial species, such as citrus, was never considered an easy task. Second, the threats to productivity and competitiveness encountered by the citrus agroindustry in Brazil require urgent and immediate solutions. Third, and perhaps the greatest challenge, was how to handle the enormous amount of information generated by an EST sequencing project. Obviously, many of these challenges still have loose ends; however, after finishing this project, new approaches become feasible and the group is still continuously working in the network.

Botanical, genetic and horticultural difficulties are the main challenges to classical breeding approaches for citrus species. To improve and to achieve faster results in citrus breeding, new technologies and approaches have been incorporated allowing better and faster acquisition of knowledge on the genetics of this group, as well as on the relationship with their pathogens. Genome information will allow understanding and incorporating more details on interactions within such complex biological systems.

Our coverage of the citrus genome is, of course, incomplete, both from the biological perspective (a number of biological paradigms in citrus still remain to be fully understood) and from the methodological side (certainly, there are many more genes than those sequenced so far). However, a number of new themes have emerged from these data. In fact, it is this what makes the next step - citrus functional genomics - a very interesting area to work in and to which more scientists are becoming attracted as a consequence of the initial proposal. Hopefully, this publication will encourage the integration of the various citrus genomics projects that are underway worldwide.

Finally, the process of preparing this special issue of GMB has revealed a high degree of cooperativeness among the authors, who shared their expertise with the major goal of exploring the genome of citrus to identify how we can benefit from it. Thus, this first publication only dedicated to the genome of citrus was capable of sharing with the scientific community over 30 articles, authored by nearly 80 scientists from several institutions that discussed the citrus genomic data regarding various features of its genetics and biology. Moreover, all ESTs used in this project will be publicly available on www.centrodecitricultura.br/citest, as well as in the GenBank database (www.ncbi.nlm.nih.gov).

The CitEST network thanks the Brazilian National Research Council (CNPq) for supporting this project within the "Millennium Institutes". 